\title{
Doxorubicin-loaded quaternary ammonium palmitoyl glycol chitosan polymeric nanoformulation: uptake by cells and organs
}

This article was published in the following Dove Press journal: International Journal of Nanomedicine

\author{
Ummarah Kanwal ${ }^{1,2}$ \\ Nadeem Irfan Bukhari \\ Nosheen Fatima Rana ${ }^{3}$ \\ Mehreen Rehman' \\ Khalid Hussain ${ }^{2}$ \\ Nasir Abbas ${ }^{2}$ \\ Arshad Mehmood ${ }^{4}$ \\ Abida Raza' \\ 'NILOP Nanomedicine Research \\ Laboratories, National Institute \\ of Lasers and Optronics, Pakistan \\ Institute of Engineering and Applied \\ Sciences Islamabad, Pakistan; \\ ${ }^{2}$ University College of Pharmacy, \\ University of the Punjab, Allama lqbal \\ Campus, Lahore, Pakistan; ${ }^{3}$ Department \\ of Biomedical Engineering and \\ Sciences, School of Mechanical and \\ Manufacturing Engineering, National \\ University of Sciences and Technology, \\ Islamabad, Pakistan; ${ }^{4}$ Material Division, \\ National Institute of Lasers and \\ Optronics, Pakistan Institute of \\ Engineering and Applied Sciences \\ Islamabad, Islamabad, Pakistan
}

Correspondence: Nadeem Irfan Bukhari University College of Pharmacy, University of the Punjab, Allama lqbal Campus, Lahore 54000, Pakistan Tel +92 3004259738

Email nadeem_irfan@hotmail.com

Abida Raza

Group Head Nanotheranostics Research, NILOP Nanomedicine Research Laboratories, National Institute of Lasers and Optronics, Pakistan Institute of Engineering and Applied Sciences, Lehtrar Road, Nilore 44000, Pakistan

Tel +92345 771 3910

Fax +92 5I 220805 I

Email abida_rao@yahoo.com
Purpose: This study was aimed to develop doxorubicin-loaded quaternary ammonium palmitoyl glycol chitosan (DOX-GCPQ) nanoformulation that could enable DOX delivery and noninvasive monitoring of drug accumulation and biodistribution at tumor site utilizing selfflorescent property of doxorubicin.

Materials and methods: DOX-GCPQ amphiphilic polymeric nanoformulations were prepared and optimized using artificial neural network (ANN) and characterized for surface morphology by atomic force microscopy, particle size with polydispersity index (PDI), and zeta potential by dynamic light scattering. Fourier transformed infrared (FTIR) and X-ray diffractometer studies were performed to examine drug polymer interaction. The ANN-optimized nanoformulation was investigated for in vitro release, cellular, tumor, and tissue uptake.

Results: The optimized DOX-GCPQ nanoformulation was anionic spherical micelles with the hydrodynamic particle size of $97.8 \pm 1.5 \mathrm{~nm}$, the PDI of $<0.3$, the zeta potential of $28 \pm 2 \mathrm{mV}$, and the encapsulation efficiency of $80 \% \pm 1.5 \%$. Nanoformulation demonstrated a sustained release pattern over $48 \mathrm{~h}$, assuming Weibull model. Fluorescence microscopy revealed higher uptake of DOX-GCPQ in human rhabdomyosarcoma (RD) cells as compared to free DOX. In vitro cytotoxicity assay indicated a significant cytotoxicity of DOX-GCPQ against RD cells as compared to DOX and blank GCPQ $(P<0.05)$. DOX-GCPQ exhibited low $\mathrm{IC}_{50}(1.7 \pm 0.404 \mu \mathrm{mol})$ when compared to that of DOX $(3.0 \pm 0.968 \mu \mathrm{mol})$. In skin tumor xenografts, optical imaging revealed significantly lower DOX-GCPQ in heart and liver $(P<0.05)$ and accumulated mainly in tumor $(P<0.05)$ as compared to other tissues.

Conclusion: The features of nanoformulation, ie, small particle size, sustained drug release, and enhanced cellular uptake, potential to target tumor passively coupled with the possibility of monitoring of tumor localization by optical imaging may make DOX-GCPQ an efficient nanotheranostic system.

Keywords: quaternary ammonium palmitoyl glycol chitosan, doxorubicin, artificial neural network, optical imaging, biodistribution, nanotheranostic

\section{Introduction}

Doxorubicin (DOX), a member of anthracycline family, is an anticancer drug used in the treatment of breast, skin, and hepatocellular cancers, lymphomas, and solid tumors. ${ }^{1,2}$ Certain issues have been associated with DOX, for instance, after intravenous administration, it undergoes hydrolytic degradation in plasma and rapidly eliminated by enzymes. ${ }^{3}$ Furthermore, despite having broad anticancer activity, the clinical use of DOX has been offset against its cardiotoxicity. ${ }^{4}$ Several efforts have been directed to address the above issues of DOX. Recently, the US Food \& Drug Administration has submit your manuscript Dovepress $f y$ in 1 
approved Doxil ${ }^{\circledR}$ (Janssen Products, LP., Horsham, PA, USA) and Myocet ${ }^{\circledR}$ (Sopherion Therapeutics, LLC, Princeton, NJ, USA), liposomal DOX nanoformulations, but they still pose toxicity problems. ${ }^{5}$ Therefore, designing DOX delivery system is required to address the limitations associated with DOX and to further increase its antitumor efficacy. Several nanoparticulate-based DOX delivery systems include polymeric nanoparticles, lipid-based nanoparticles, inorganic nanoparticles, nanogels, nanotubes, and nanocrystals, which are in preclinical phase. ${ }^{6-10}$ Liposomal DOX uptake by reticuloendothelial system (RES) prevents its delivery at tumor site with reduced efficacy. ${ }^{11,12}$ Nanomedicine, an excipient-free system, is a promising approach to overcome nonselective distribution of chemotherapeutic drugs associated with conventional therapy.$^{13}$ Among the above delivery systems, the polymeric DOX nanoformulations are more promising in achieving enhanced efficacy with lesser side effects and reduced cytotoxicity by targeting DOX at specific site..$^{14,15}$ Polymeric formulations also exhibit extended half-life and decreased uptake by liver and demonstrate enhanced permeability and retention (EPR) effect. ${ }^{16}$

An amphiphilic polymer quaternary ammonium palmitoyl glycol chitosan (GCPQ) was used in the present study, which forms stable micelles as DOX carrier. GCPQ self-assembles at the neutral $\mathrm{pH}$ due to its 6-O glycol unit, which enables GCPQ to form micelles and is presented as low-viscosity dispersion (dynamic viscosity $<5 \mathrm{mPa}$ ). The GCPQ dispersion sustains its form as long as its concentration remains below $40 \mathrm{mg} / \mathrm{mL}$. The above features make GCPQ favorable for injectable with expanded utility. In addition, GCPQ does not exhibit liver and spleen uptake. ${ }^{17}$

The functionality of polymeric nanoparticles can be enhanced by the use of fluorescent ligand, thus making optical imaging possible for the distributed nanoparticles in tissues, organs, and tumors. ${ }^{18}$ Optical imaging can monitor the biodistribution of nanomedicine noninvasively for early cancer detection and the monitoring of cancer therapy. ${ }^{19}$ Fluorescent polymeric nanoparticles at cellular and organ levels have already been utilized for in vivo imaging of tumor tissues..$^{20}$ DOX's auto-fluorescence features (such as florescence absorption and excitation wavelengths of 480 and $590 \mathrm{~nm}$, respectively) enable the monitoring of its biodistribution to tissues by optical imaging in addition to its anticancer activity, which has made it attractive for cancer research. ${ }^{21,22}$ Hence, the aim of present study was to prepare DOX-GCPQ as dual property optical drug delivery system (ODDS) without the use of chemical cross-linkers such as glutaraldehyde ${ }^{23}$ and ionic gelation agents, ie, sodium tripolyphosphate, to minimize the side effects associated with excipients..$^{24}$ ODDS could deliver DOX at tumor site without nonspecific uptake by heart, liver, and RES and monitored for tissue uptake and tumor accumulation through in vivo optical imaging using a skin tumor model.

\section{Materials and methods Materials}

Glycol chitosan (GC) (G7753), sodium iodide (383112), methyl iodide (67692), sodium bicarbonate (792519), palmitic acid $N$-hydroxysuccinimide (P1162), $N$-methyl-2pyrrolidone (1437202), PBS tablets (P 4417), cholesterol (C8667), pyrene (185515), acetone (65050), DOX (44583), 7,12-dimethylbenz(a)anthracene (DMBA) (D3254), 12-O-tetradecanoylphorbol 13-acetate (TPA) (P1585), and solvents were supplied by Sigma-Aldrich Co. (St Louis, MO, USA). Sephadex G-25 was purchased from GE Healthcare BioSciences AB (Uppsala, Sweden). Human rhabdomyosarcoma (RD) cells ATCC CCL 136 were received as gift courtesy from National Institute of Health (NIH) (Islamabad, Pakistan). The use of RD cells was approved by Institutional Ethical Committee, University College of Pharmacy, University of Punjab (protocol no HEC/PUCP/1943A-Dated 10-4-16).

\section{Animals}

Balb/c mice of age 7-9 weeks and weight $24-28$ g purchased from NIH were used for this study. Prior to study, an ethical approval was obtained from the Animal Ethics Committee, University College of Pharmacy, University of Punjab (protocol no AEC/PUCP/1059A-dated 28-5-16). All the experiments in animals in this work were undertaken in accordance with the International Conference on Harmonization ICH guidelines. ${ }^{25}$

\section{Methods}

\section{Synthesis and characterization of nanocarrier}

GCPQ was synthesized by the acid degradation of GC followed by covalent coupling of palmitic acid $N$-hydroxysuccinimide (palmitoylation), and quaternization was achieved by methylation with methyl iodide using a previously reported quaternization method. ${ }^{26,27}$ Briefly, $500 \mathrm{mg}$ of hydrolyzed GC and $376 \mathrm{mg}$ of sodium bicarbonate added in flask already containing water:ethanol solution $(76: 24 \mathrm{~mL})$. Palmitic acid $\mathrm{N}$-hydroxysuccinimide solution was added in GC and sodium bicarbonate solution under continuous stirring for $72 \mathrm{~h}$. Product was separated via the evaporation of ethanol and redispersed in distilled water. This mixture was extracted three times with ample diethyl ether to remove any excess of palmitic acid 
$N$-hydroxysuccinimide. The mixture was dialyzed (molecular weight cutoff $12-14 \mathrm{kDa}$ ) against $5 \mathrm{~L}$ of distilled water for $24 \mathrm{~h}$ with six changes. The dialysate was freeze dried. Quaternization was carried out by dispersing the palmitoyl GC (300 mg) in $25 \mathrm{~mL}$ of $N$-methyl-2 pyrrolidone for $12 \mathrm{~h}$. Ethanolic solution $(5 \mathrm{~mL})$ was prepared by dissolving sodium hydroxide (40 mg) and sodium iodide (45 mg) and added to palmitoyl GC solution. Finally, methyl iodide ( $1 \mathrm{~g}$ ) was added and kept under nitrogen gas for $3 \mathrm{~h}$ at $36^{\circ} \mathrm{C}$ in dark. Diethyl ether was used to precipitate quaternary ammonium product and was washed twice with the mixture of diethyl ether and absolute ethanol and redispersed in water. The dialysate (iodide salt) was purified through Amberlite-96 resins. Sodium chloride $(0.1 \mathrm{M})$ and sodium bicarbonate $(0.01 \mathrm{M})$ were utilized for the dialysis of clear eluent up to $4.5 \mathrm{~h}$ with three changes. Afterward, $5 \mathrm{~L}$ of water was used for extensive dialysis for $24 \mathrm{~h}$ with six changes. Finally, GCPQ was obtained as white cotton-like solid after freeze drying. The level of modification of GCPQ after palmitoylation and quaternization was confirmed by $\mathrm{NMR}^{27}$ (400 MHz on Bruker AMX spectrometer; Bruker AXS Inc., Madison, WI, USA). GCPQ polymer was used for the preparation of DOX nanoformulations.

\section{Preparation of DOX-GCPQ nanoformulations}

Fixed amount of GCPQ polymer $(5 \mathrm{mg} / \mathrm{mL})$ was loaded with varying concentrations of DOX ranging from 2 to $6 \mathrm{mg} / \mathrm{mL}$ according to Table 1 in normal saline by using ultrasonic probe-sonicator (Vibra Cell; Sonics, Newtown, CT, USA) operating at varying amplitudes and times as shown in Table 1. For sonication, a probe of diameter $6 \mathrm{~mm}$ was put in the middle of the light-protected $2 \mathrm{~mL}$ sample placed in ice and sonicated at pulse mode of $50 \mathrm{~s}$ on and $10 \mathrm{~s}$ off positions.

\section{Characterization of DOX nanoformulation}

\section{Measurement of size, PDI, and zeta potential}

Hydrodynamic diameter, polydispersity index (PDI) value and zeta potential of drug loaded nanoformulations were

Table I Composition and operating conditions for formulation of DOX-GCPQ

\begin{tabular}{|l|l|l|l|}
\hline Experiments & Drug $(\mathbf{m g})$ & Amplitude (\%) & Time (min) \\
\hline 1 & 2 & 80 & 7 \\
\hline 2 & 3 & 75 & 8 \\
\hline 3 & 4 & 65 & 8 \\
\hline 4 & 5 & 55 & 10 \\
\hline 5 & 6 & 60 & 10 \\
\hline
\end{tabular}

Abbreviations: DOX, doxorubicin; GCPQ, quaternary ammonium palmitoy glycol chitosan. analyzed by dynamic light scattering (DLS). Analysis was done in triplicate at $25^{\circ} \mathrm{C}$ (Microtrac Nanotrac Wave II, USA). Sample for atomic force microscopy (AFM) analysis was prepared by drying nanoformulation at $25^{\circ} \mathrm{C}$ for period of two days in desiccator containing dried silica gel. Surface morphology was studied by acquiring the images on digital AFM instrument at non-contact mode (Alpha Contec, Germany) equipped with silicon cantilever of $7 \mathrm{~nm}$ in $5 / 5 \mu \mathrm{m}$ scan area size.

\section{Encapsulation efficiency}

Nonencapsulated DOX from nanoformulation (DOXGCPQ) was separated by size exclusion chromatography using Sephadex column G-25. Encapsulation efficiency was calculated according to the following equation:

$$
\mathrm{EE} \%=\frac{\mathrm{Q}_{\mathrm{t}}-\mathrm{Q}_{\mathrm{r}}}{\mathrm{Q}_{\mathrm{t}}^{*}} \times 100
$$

where $Q_{t}$ is the total drug contained in the formulation and $\mathrm{Q}_{\mathrm{r}}$ is free DOX.

Calibration curve of DOX was constructed, and its encapsulation efficiency was calculated by measuring the absorbance of both DOX-GCPQ and DOX at $485 \mathrm{~nm}$ using UV-Vis spectrophotometer (Model U-2900; Hitachi Ltd., Tokyo, Japan).

\section{Optimization of DOX nanoformulations}

The data of properties for five nanoformulations were used for formulation optimization, using artificial neural network (ANN) approach by employing Neural-Power, Ver 3.1 (CPC-X Software, USA). For employing ANN-based optimization, drug concentration, polymer amount, sonication amplitude, and sonication time were taken as the input parameters, while size, PDI, zeta potential, loading efficiency, and their respective SD were measured as outputs using ANN architecture (Figure 1).

Quick propagation method was used with Tanh as transfer function. The input, output, and hidden layer were set as 4, 8, and 1 , respectively, with the number of nodes 8 . The aspired criteria for output responses were used as follows: size $<100 \mathrm{~nm}$, PDI $<1$, zeta potential $\geq 25 \mathrm{mV}$, and drug loading $80 \%$ while SD of all above outputs was set as minimum (ie, 0). Based on the desirability levels of the outputs, ANN approach yields forecasted composition and conditions for the optimized formulation, also with forecasted levels of outputs. ${ }^{18}$ ANN-generated forecasted inputs (composition and 


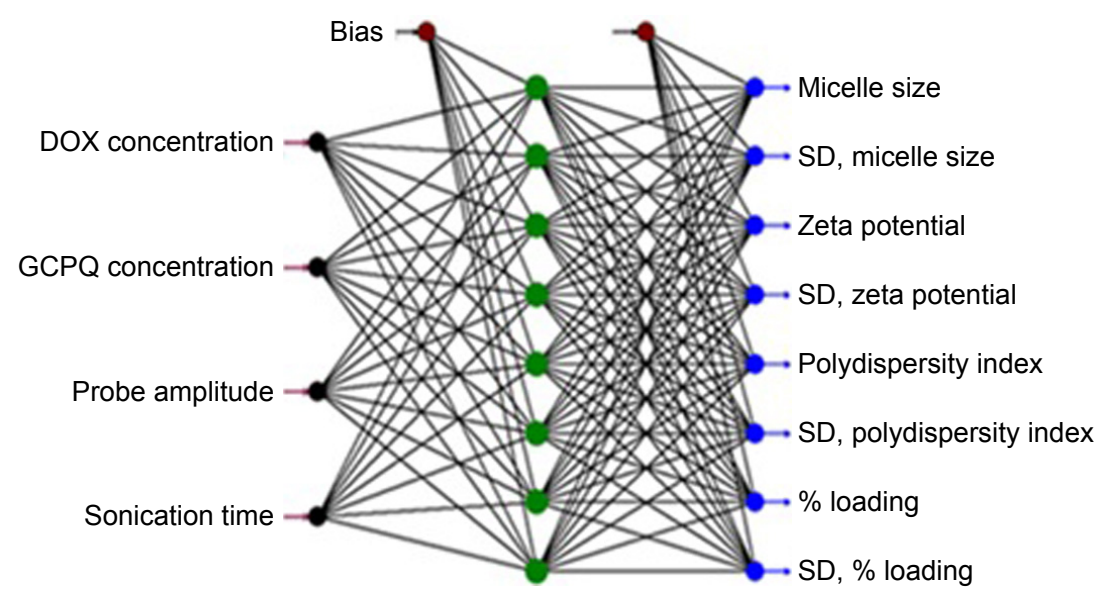

Figure I Architect of artificial network employed for the optimization of DOX formulation using GCPQ.

Abbreviations: DOX, doxorubicin; GCPQ, quaternary ammonium palmitoyl glycol chitosan.

conditions) were used to produce a confirmation formulation which was characterized again.

\section{Characterization of optimized (confirmation) formulation}

The optimized formulation was characterized for size, PDI, zeta potential, surface morphology, and percentage of drug loading using the same procedures stated earlier. The optimized nanoformulation was subjected to the additional in vitro and in vivo tests such as Fourier transformed infrared (FTIR), X-ray diffractometer (XRD), in vitro drug release, cellular uptake, in vitro cytotoxicity, and in vivo tumor and tissue uptake studies.

\section{FTIR and XRD studies}

Drug-polymer interaction for the optimized DOX nanoformulation was studied through FTIR spectroscopy. FTIR spectra were recorded in mid-IR range $(4,000-400 / \mathrm{cm}$ using FTIR Spectrophotometer, equipped with software OMNIC ${ }^{\text {TM }}$ Version 6.0 a; Thermo Fisher Scientific, Waltham, MA, USA). FTIR spectra of DOX, polymer (GCPQ), and optimized DOX-GCPQ nanoformulation were compared with each other to find the interaction of drug with polymer. Freeze-dried DOX-GCPQ nanoformulation was subjected to XRD studies. Encapsulation of drug-loaded polymeric nanoformulation, its crystallographic properties, and physical state was analyzed by X-ray diffractometer (Bruker AXS Inc.). For XRD, $2 \vartheta$ angle was measured from 0 to $1,000^{\circ} \mathrm{C}$ at the scanning speed of $2^{\circ} \mathrm{C} / \mathrm{min}$ and $\mathrm{X}$-ray radiation source was copper at $40 \mathrm{kV}$ with $40 \mathrm{~mA}$.

\section{In vitro drug release studies}

DOX release from optimized DOX-GCPQ was determined by dialysis membrane diffusion technique. Nanoformulation $(1 \mathrm{~mL})$ was dialyzed against $200 \mathrm{~mL}$ of dissolution medium, PBS, $\mathrm{pH} 7.4$, using the dialysis membrane (molecular weight cutoff $12 \mathrm{kDa}$ ). Release pattern was compared with the DOX solution. Studies were conducted at $37^{\circ} \mathrm{C} \pm 0.5^{\circ} \mathrm{C}$ with continuous stirring at $50 \mathrm{rpm}$ for $48 \mathrm{~h}$. Samples were collected at predetermined time intervals, ie, at $10 \mathrm{~min}$, and then on $0.5,1.0,1.5,2.0,2.5,3.0,4.0,6.0,8.0$, $12.0,16.0,20.0,24.0$, and $48.0 \mathrm{~h}$ for the determination of drug concentration by UV-Vis spectrophotometer (Model U-2900) at $485 \mathrm{~nm} .{ }^{28}$ Kinetic modeling of drug release was assessed by the DD-Solver software by entering the percent release data of DOX and DOX-GCPQ. Different release kinetics models (Table 2) were employed, the best model was selected based on the highest value of coefficient of determination $\left(R^{2}\right)$, and in case of a close $R^{2}$ value for two models, the lowest value of Akaike information criterion (AIC) was used. To understand the release mechanism, $n$ value for DOX-GCPQ was computed using the Korsmeyer-Peppas model.

The dissimilarity factor $\left(f_{1}\right)$ and similarity factor $\left(f_{2}\right)$ were also computed between the free and optimized DOX nanoformulation with the equation given. ${ }^{29}$

$$
\begin{gathered}
f_{1}=\left[\frac{\sum_{t=1}^{n}\left(R_{t}-T_{t}\right)}{\sum_{t=1}^{n} R_{t}}\right] \times 100 \\
f_{2}=50 \times \log \left[\left\{1+\left(\frac{1}{n}\right) \sum_{t=1}^{n}\left(R_{t}-T_{t}\right)^{2}\right\}^{-0.5} \times 100\right]
\end{gathered}
$$

where $\mathrm{n}=$ sample number, $\mathrm{R}_{\mathrm{t}}=$ percentage of reference, and $T_{t}=$ percentage of test drug release.

\section{Cellular uptake studies}

Utilizing DOX self-fluorescent property with excitation wavelength 480 and $560 \mathrm{~nm}$, cellular uptake of the optimized 
Table 2 DOX-GCPQ release kinetics models employed in study

\begin{tabular}{|c|c|}
\hline Model & Equation \\
\hline Zero order' & $\begin{array}{l}\qquad Q_{t}=K_{0} t \\
\text { where } Q_{t}=\text { drug amount dissolved, } t=\text { time, and } \\
\left.K_{0}=\text { zero-order release constant (concentration } / \mathrm{t}\right)\end{array}$ \\
\hline First order"l & $\begin{array}{l}\qquad Q_{t}=\ln Q_{0}-K_{1} t \\
\text { where } Q_{t}=\text { cumulative drug amount release, } t=\text { time, } \\
\text { and } K=\text { first-order release constant }\end{array}$ \\
\hline Higuchi' $^{12}$ & $\begin{array}{l}\qquad Q=K_{H} t^{\frac{1}{2}} \\
\text { where } Q=\text { drug amount released per unit area in } \\
\text { time } t \text { and } K_{H}=\text { Higuchi dissolution constant }\end{array}$ \\
\hline $\begin{array}{l}\text { Korsmeyer- } \\
\text { Peppas }^{29}\end{array}$ & $\begin{array}{l}\qquad M_{t} / M_{\infty}=K_{t^{n}} \\
\text { where } M_{t} / M_{\infty}=\text { fraction of drug released at time } t \text {, } \\
k=\text { release rate constant, and } n=\text { value to } \\
\text { characterize different release }\end{array}$ \\
\hline $\begin{array}{l}\text { Hixson- } \\
\text { Crowell }^{30}\end{array}$ & $\begin{array}{l}\qquad \mathrm{W}_{0} \frac{\mathrm{l}}{3}-\mathrm{W}_{\mathrm{t}} \frac{\mathrm{l}}{3}=\kappa \mathrm{t} \\
\text { where } \mathrm{W}_{0}=\text { initial drug amount, } \mathrm{W}_{\mathrm{t}}=\text { remaining } \\
\text { drug amount at time } t \text {, and } \mathrm{\kappa}(\mathrm{kappa})=\text { constant } \\
\text { incorporating surface-volume relation }\end{array}$ \\
\hline $\begin{array}{l}\text { Weibull } \\
\text { model }^{22}\end{array}$ & $\begin{array}{l}\qquad \log [-\ln (I-m)]=b \log \left[t-T_{i}\right]-\log a \\
\text { where } a=\text { time dependence, } b=\text { dissolution curve } \\
\text { shape, and } m=\text { drug amount dissolved }\end{array}$ \\
\hline
\end{tabular}

Abbreviations: DOX, doxorubicin; GCPQ, quaternary ammonium palmitoyl glycol chitosan.

DOX-GCPQ nanoformulation was analyzed by fluorescence microscopy. Human RD cells were seeded at the density of $1.0 \times 10^{5}$ cells/well in six-well plate (Corning Incorporated, Corning, NY, USA) and allowed to grow for $24 \mathrm{~h}$. Three wells of cells were exposed for each DOX and DOX-GCPQ for $6 \mathrm{~h}$, and experiment was conducted in triplicate. Cells, washed thrice with PBS, were imaged with fluorescence microscopy using GFP filter $\left(\right.$ Evos $^{\circledR}$ FL Cell Imaging System; Thermo Fisher Scientific). The untreated cells served as control. ${ }^{31}$

\section{Determination of in vitro cytotoxicity and $\mathrm{IC}_{50}$}

Cytotoxicity of the optimized DOX-GCPQ nanoformulation was evaluated by the MTT assay against RD cell line. Cells, $1.0 \times 10^{4} /$ well, were seeded in 96-well plate (Corning Incorporated) and allowed to grow by incubating for $24 \mathrm{~h}$ at $37^{\circ} \mathrm{C}$ in $5 \% \mathrm{CO}_{2}$ at $85 \%$ humidity incubator (Model NU 5700; Nuaire, Plymouth, MA, USA). After 24 h, the culture medium was removed and, to calculate $\mathrm{IC}_{50}$, three wells of cells were incubated with concentrations ranging from 0.01 , $0.08,0.40,0.80,1.30,1.70,2.10,2.50,3.0,3.40,3.80$, and $4.30 \mu \mathrm{mol}$ of GCPQ, DOX, and DOX-GCPQ for 24, 48, and $72 \mathrm{~h}$ at $37^{\circ} \mathrm{C}$. The drug solution and formulation were removed, MTT was added in each well, and cells were incubated for $4 \mathrm{~h}$ at $37^{\circ} \mathrm{C}$. The solution was aspirated, and the resulting formazan crystals were dissolved in dimethyl sulfoxide. Microplate reader (Model FL $\times 800$; Biotek, Winooski, VA, USA) was used to measure absorbance at $485 \mathrm{~nm} .{ }^{21,32}$ Cytotoxicity was expressed as percentage of cell viability compared to untreated control cells. $\mathrm{IC}_{50}$ was calculated using the regression analysis implemented in GraphPad Prism Ver 7.33

\section{Tumor and tissue uptake studies using optical} imaging technique

Induction of tumor in Balb/c mice

Prior to tumor uptake study, tumor was induced in Balb/c mice using a reported method. ${ }^{34}$ Briefly, in tumor initiation phase, $0.2 \mathrm{~mL}$ of $25 \mathrm{nmol}$ solution of DMBA was applied at the dorsal skin of Balb/c mice. After 2 weeks, 1.7, 3.4, and $6.8 \mathrm{nmol}$ of 12-O-tetradecanoylphorbol 13-acetate (TPA) in $0.2 \mathrm{~mL}$ of acetone were applied to mice for tumor promotion up to 5 weeks. Mice were monitored for the development of papillomas.

\section{Administration of DOX and DOX-GCPQ nanoformulation to mice}

After 10 days of tumor implantation, when tumor volume of mice reached $50 \mathrm{~mm}^{3}$, mice were divided into two groups $(n=6)$. Group I and Group II were dosed with DOX and DOX-GCPQ nanoformulations at the final concentration of $6 \mathrm{mg} / \mathrm{kg}^{35-37}$ via tail vein. Mice were sacrificed after $24 \mathrm{~h}$ of dose administration. Tumor and vital organs including liver, kidney, heart, and spleen were harvested for tumor and tissue uptake studies by fluorescence imaging.

\section{Fluorescence imaging}

The fluorescence intensity of the ANN-optimized DOXGCPQ nanoformulation in tumor and the excised organs (heart, liver, spleen, and kidney) were examined using the iBox Explorer2 (iBox ${ }^{\circledR}$ Explorer $^{2}$ Imaging Microscope; UVP Ltd., Cambridge, UK). System was set on 535/45 excitation filter and 605/50 emission filter with the automated BioLite $^{\mathrm{TM}}$ MultiSpectral Light Source. Images were taken at $0.17 \times$ magnification keeping intensity at 6 using the $3.2 \mathrm{MP}$ OptiChemi 610 camera, Vision Works ${ }^{\circledR}$ LS Acquisition, and analysis software was used to study images.

\section{Statistical analysis}

All the experiments were conducted in triplicate, and data were presented as mean $\pm \mathrm{SD}$ or in the form specified otherwise. In vitro cytotoxicity, as measured by the percentage of cell viability, of free DOX, DOX-GCPQ, and GCPQ 
at different concentrations was compared using two-way ANOVA. Similarly, the fluorescence intensity between DOX and DOX-GCPQ treatments within different organs was also compared using two-way ANOVA with GraphPad Prism 7.0. A $P$-value of $<0.05$ was considered statistically significant difference.

\section{Results \\ GCPQ characteristics}

The GCPQ synthesized in this study demonstrated palmitoylation and quarternization levels of $34.2 \% \pm 5.188 \%$ and $8.23 \% \pm 2.4 \%$, respectively. Similarly, NMR also showed all peaks as ( $\left.\mathrm{CD}_{3} \mathrm{OD}, \mathrm{D}_{2} \mathrm{O}-9: 1\right) \delta=0.89-0.90\left(\mathrm{CH}_{3}\right.$ [palmitoyl]), $\delta=1.30\left(\mathrm{CH}_{2}\right.$ [palmitoyl] $), \delta=1.65\left(\mathrm{CH}_{2}\right.$ [palmitoyl $\beta$ to amino group]), $\delta=2.05$ ( $\mathrm{CH}_{3}$ [acetyl-glycol chitosan]), $\delta=2.2-2.4$ ( $\mathrm{CH}_{2}$ [palmitoyl $\alpha$ to amino group]), $\delta=2.7-3.2$ (proton at $\mathrm{C}_{2}$ on the sugar ring and $\mathrm{CH}_{3}-\mathrm{N}$ [dimethyl-amino-glycol chitosan]), $\delta=3.45\left(\mathrm{CH}_{3}-\mathrm{N}^{+}\right.$[trimethyl-amino-glycol chitosan] $), \delta=3.5-4.5$ (sugar protons), $\delta=3.30$ (methanol protons), $\delta=4.5-5.0$ (water protons), and $\delta=5.0$ (CH [C1 sugar proton, GC]) (Figure 2).

\section{Characteristics of nanoformulations}

Hydrodynamic particle size of DOX-GCPQ nanoformulations with zeta potential, PDI measured by nanosizer, and percentage of loading is given in Table 3 .

The data given in Table 3 were analyzed using NeuralPower, ver 3.1. The combined relative effects of probe amplitude, concentration of drug, and sonication time were shown to be $31.88,28.14$, and $29.79 \%$, respectively, on size, size distribution, zeta potential, and loading efficiency, while the contribution of polymer concentration for nanoformulation properties was only $10.18 \%$, as shown in Figure 3.

According to desirability criteria, size $<100 \mathrm{~nm}$ with PDI $<1$, zeta potential $\geq 25 \mathrm{mV}$, and loading $80 \%$, the forecasted optimized levels of the factors along with the predicted properties, generated by Neural Power ${ }^{\circledR}$, are given in Table 4. Optimized composition 1 in Table 4 (drug-topolymer ratio of $6: 5 \mathrm{mg}$ ), sonication amplitude of $60 \%$ for 10 min was selected for the preparation and characterization of a validation DOX-GCPQ formulation.

Response surface plots of outputs (properties/responses) for combined effect of inputs were generated, and the ones with paramount effect have been given herein (Figure 4). The response surface plots supported the effects as revealed in Figure 4.

Hydrodynamic particle size of the optimized DOXGCPQ nanoformulation prepared using the forecasted optimized levels of formulative ingredients and process conditions was $97.8 \pm 1.5 \mathrm{~nm}$ with the zeta potential of $-28 \pm 2 \mathrm{mV}$ and the PDI of $<0.3$ measured by nanosizer. The AFM showed that DOX-GCPQ particles were compact, spherical having smooth surface with an average diameter of $73.3 \mathrm{~nm}$ (Figure 5). The properties of the validation (optimization) formulation were comparable to the aspired as well as the ANN predicted properties of Set 1 (Table 4).

\section{FTIR spectra}

The spectrum of DOX indicated characteristics peaks/ bands at 3,560/cm (stretch, alcohol), 3,311.6/cm (OH and $\mathrm{NH}$, stretch), 2,894.3/cm (CH stretch, aromatic), 1,730/cm $(\mathrm{C}=\mathrm{O}$ stretch, ketone $), 1,630 / \mathrm{cm}(\mathrm{C}=\mathrm{O}$ stretch $), 1,282.7 / \mathrm{cm}$ (C-O-C, stretch), 1,114/cm (C-O stretch, tertiary alcohol), 1,070/cm (C-O stretch, secondary alcohol), and 988/cm (C-O stretch, primary alcohol). In contrast, the spectrum of DOX-GCPQ indicated that peaks/bands at 3,560/cm (stretch, alcohol), 1,114/cm (tertiary alcohol), 1,070/cm (secondary alcohol), and 988/cm (primary alcohol) were disappeared and the peak corresponding $\mathrm{OH}$ and $\mathrm{NH}$ was broadened.

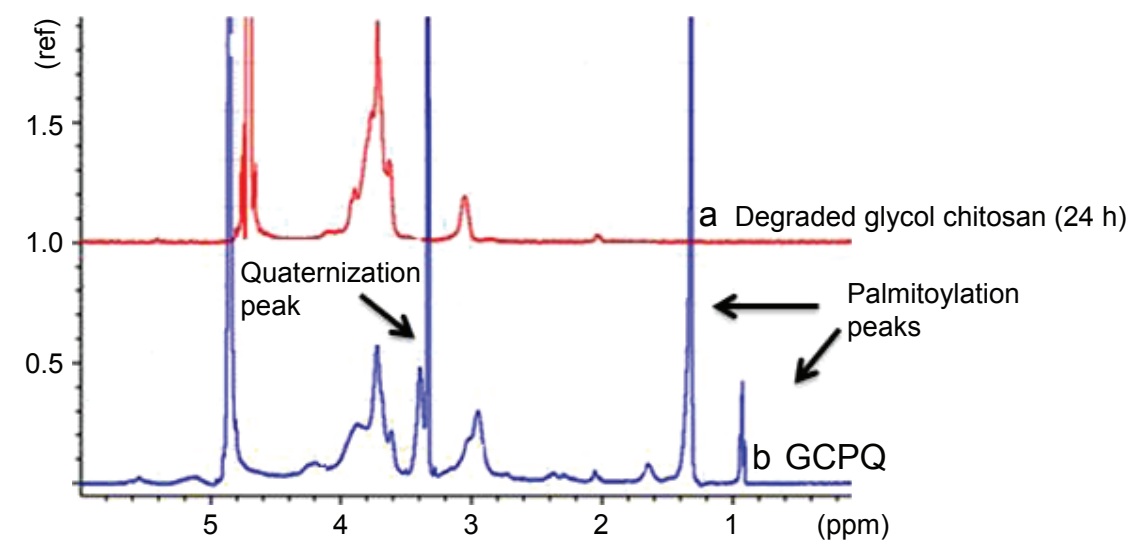

Figure 2 NMR spectrum of (a) degraded glycol chitosan and (b) GCPQ. Abbreviation: GCPQ, quaternary ammonium palmitoyl glycol chitosan. 
Table 3 Characteristics of the doxorubicin nanoformulations

\begin{tabular}{|c|c|c|c|c|c|c|c|c|c|c|c|}
\hline \multirow[t]{2}{*}{ Experiments } & \multirow[t]{2}{*}{$\begin{array}{l}\text { Drug } \\
\text { (mg) }\end{array}$} & \multirow[t]{2}{*}{ Amplitude (\%) } & \multirow[t]{2}{*}{$\begin{array}{l}\text { Time } \\
\text { (min) }\end{array}$} & \multicolumn{2}{|c|}{ Size $(\mathrm{nm})$} & \multicolumn{2}{|c|}{$\begin{array}{l}\text { Zeta potential } \\
(\mathrm{mV})\end{array}$} & \multicolumn{2}{|l|}{ PDI } & \multicolumn{2}{|c|}{ Loading (\%) } \\
\hline & & & & Mean & SD & Mean & SD & Mean & SD & Mean & SD \\
\hline I & 2 & 80 & 7 & 298.2 & 7.2 & 55 & 2.2 & 0.855 & 0.037 & 16.04 & 0.33 \\
\hline 2 & 3 & 75 & 8 & 129.2 & 4.5 & 47 & 4.5 & 0.602 & 0.042 & 28.22 & 0.24 \\
\hline 3 & 4 & 65 & 8 & 148.2 & 2.2 & 40 & 1.0 & 0.900 & 0.101 & 61.02 & 2.09 \\
\hline 4 & 5 & 55 & 10 & 120.4 & 0.2 & 35 & 3.5 & 0.622 & 0.102 & 74.30 & 0.61 \\
\hline 5 & 6 & 60 & 10 & 97.8 & 1.5 & 28 & 2.0 & 0.322 & 0.220 & 81.22 & 1.04 \\
\hline
\end{tabular}

Abbreviation: PDI, polydispersity index.

The band of $\mathrm{CH}$ stretch remained intact whereas the peak intensity of carbonyl group was reduced as shown in Figure 6.

\section{XRD spectra of DOX and DOX-GCPQ nanoformulation}

XRD spectra of DOX and DOX-GCPQ nanoformulation (Figure 7) showed that the peaks were obtained at the $2 \theta$ of $11.45,13.12,18.76,22.45,25.42,31.24$, and 38.94 for crystalline DOX, whereas these peaks disappeared in case of DOX-GCPQ nanoformulation, based on which the DOX in nanoformulation was considered to be amorphous. Furthermore, it supported a successful encapsulation of DOX inside polymer.

\section{DOX-GCPQ in vitro release kinetics}

As shown in Figure 8, approximately all of free DOX $(100 \%)$ diffused out of dialysis membrane into buffer within $10 \mathrm{~h}$. DOX release rate from DOX-GCPQ nanoformulation was considerably slow at $\mathrm{pH} 7.4$. Only $35 \%$ of drug came out in the medium within $24 \mathrm{~h}$ (Figure 8). Drug release from nanoformulation followed Weibull model based on $R^{2}$ and AIC values as shown in Table 5. The parameters of Weibull model, ie, $a$ and $b$, were found to be 12.34 and 0.63 , respectively. The $n$ value, based on Korsmeyer-Peppas was 0.59 .

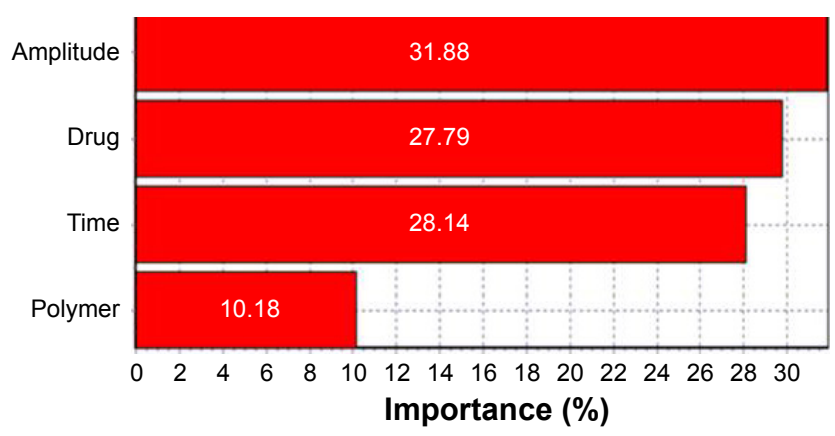

Figure 3 Percentage effect of input factors on DOX-GCPQ formulation. Abbreviations: DOX, doxorubicin; GCPQ, quaternary ammonium palmitoyl glycol chitosan.

\section{Cellular uptake of DOX-GCPQ}

After $6 \mathrm{~h}$ incubation of RD cells against DOX and DOXGCPQ, fluorescence images (Figure 9) showed an enhanced fluorescence intensity of DOX-GCPQ as compared to free DOX.

\section{In vitro cytotoxicity}

DOX and DOX-GCPQ nanoformulations showed concentration-dependent toxicity at $24 \mathrm{~h}$ (Figures 10 and 11). DOX-GCPQ nanoformulation indicated significant cytotoxic effect after $24 \mathrm{~h}$ to RD cell as compared to DOX and GCPQ alone $(P<0.05)$. The $\mathrm{IC}_{50}$ value of DOX was found to be $3.0 \pm 0.968 \mu \mathrm{mol}$ as compared to $1.7 \pm 0.404 \mu \mathrm{mol}$ of DOXGCPQ nanoformulation at $24 \mathrm{~h}$.

\section{Biodistribution}

Free DOX showed nonsignificantly higher $(P>0.05)$ uptake in liver as compared to DOX-GCPQ. In heart and spleen, the biodistribution of DOX-GCPQ was significantly lower $(P<0.05)$ as compared to free DOX. DOX-GCPQ with high fluorescent signal accumulated in tumor tissue in comparison to the other organs such as heart and liver (Figure 12). When tumor uptake of DOX-GCPQ was compared to that of all organs, significantly higher distribution was noted $(P<0.05)$, except for the kidney $(P>0.05)$.

\section{Discussion}

Currently, there is a need of developing such anticancer therapy, which can successfully deliver the anticancer drugs to the tumors, as well as simultaneously enable noninvasive assessment of therapy outcome by monitoring its tumor localization/biodistribution. ${ }^{38}$ The multifunctional nanoparticles attached with fluorescent ligand or containing a self-fluorescent drug can serve this purposes. ${ }^{39}$ The present study was aimed to develop DOX-GCPQ nanoformulation that could enable drug delivery and noninvasive monitoring of tumor accumulation and biodistribution, 
Table 4 Forecasted optimized compositions of the formulations factors and predicted responses based on optimized compositions and conditions

\begin{tabular}{|c|c|c|c|c|c|c|}
\hline \multirow[t]{2}{*}{ Inputs } & \multirow[t]{2}{*}{ Desirability } & \multicolumn{5}{|c|}{ Optimized compositions/conditions } \\
\hline & & 1 & 2 & 3 & 4 & 5 \\
\hline Drug (mg) & - & 6 & 6 & 6 & 5.6 & 5.6 \\
\hline Polymer (mg) & - & 5 & 5 & 5 & 5 & 5 \\
\hline Amplitude (\%) & - & 57.5 & 57.5 & 60 & 60 & 62.5 \\
\hline Time (s) & - & 10 & 9.7 & 9.4 & 10 & 10 \\
\hline Outputs & & \multicolumn{5}{|c|}{ Predicted responses with above composition/conditions } \\
\hline Size $(n m)$ & $<100$ & 99.86 & 100.23 & 100.16 & 100.83 & 99.46 \\
\hline Size (SD) & $\approx 0$ & 1.11 & 1.25 & 1.83 & 1.18 & 1.56 \\
\hline Zeta $(\mathrm{mV})$ & $\geq 25$ & 28.50 & 28.48 & 28.25 & 28.69 & 28.32 \\
\hline Zeta (SD) & $\approx 0$ & 2.43 & 2.16 & 1.46 & 2.08 & 1.67 \\
\hline PDI & $\approx 0$ & 0.34 & 0.35 & 0.36 & 0.35 & 0.34 \\
\hline PDI (SD) & $\approx 0$ & 0.212347 & 0.213498 & 0.219284 & 0.211818 & 0.218289 \\
\hline Loading (\%) & $>80$ & 80.58154 & 80.50128 & 80.73271 & 80.24524 & 80.76807 \\
\hline Loading (SD) & $\approx 0$ & 0.817227 & 0.961826 & 1.493892 & I.08887| & 1.379792 \\
\hline Sum of abs error & - & 9.136 & 9.1464 & 9.5115 & 9.661 & 9.7912 \\
\hline
\end{tabular}

Abbreviation: PDI, polydispersity index.

utilizing self-florescent property of DOX. For this purpose, hydrophobically modified amphiphilic GC (GCPQ) was synthesized by the acid degradation of GC, followed by covalent coupling of palmitic acid called palmitoylation and quarternization of GC as reported. ${ }^{26,27}$ The palmitoylation level of $34.2 \% \pm 5.188 \%$ was a reason for negative charge, while the quaternization level of $8.23 \% \pm 2.4 \%$ was a reason for positive charge. The synthesized GCPQ was able to self-assemble at low critical micelle concentration (CMC) into particles of colloidal size that also indicated its

A
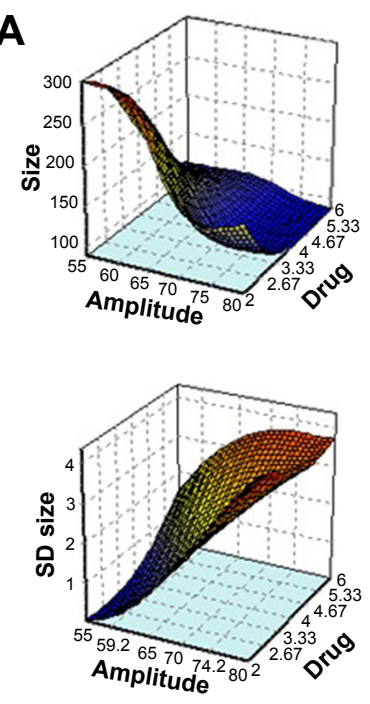
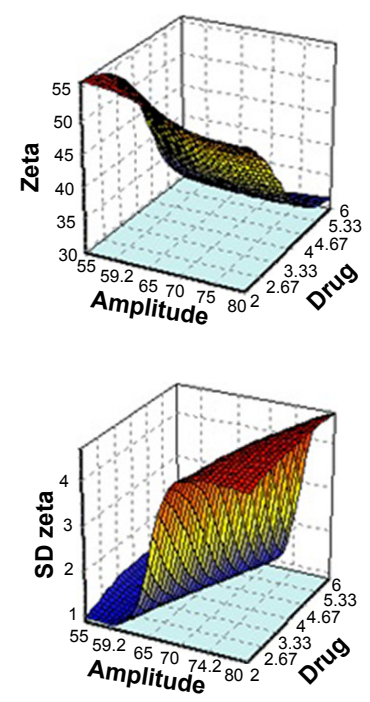

colloidal stability. ${ }^{40}$ DOX was loaded into GCPQ by probe sonication. To achieve the optimized GCPQ nanoparticle with desired features such as size $<100 \mathrm{~nm}$, PDI value $<1$, zeta potential $\geq 25 \mathrm{mV}$, and loading $80 \%$, parameters such as drug amount, polymer amount, sonication speed, and the sonication parameters were manipulated since these factors have reported to affect the size of the nanoparticles. ${ }^{41}$ ANN provides relative importance, as percentage contribution of different inputs for overall properties, ${ }^{42}$ which along with response surface plots help in finding the optimum levels of

Figure 4 (Continued)
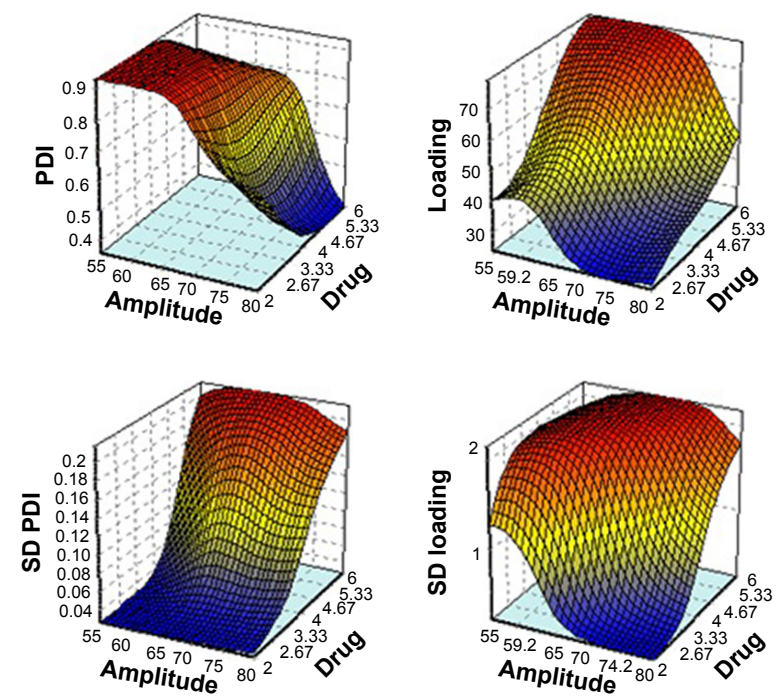
B
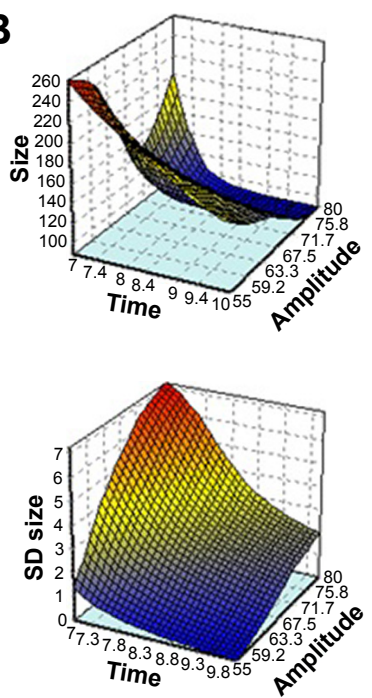
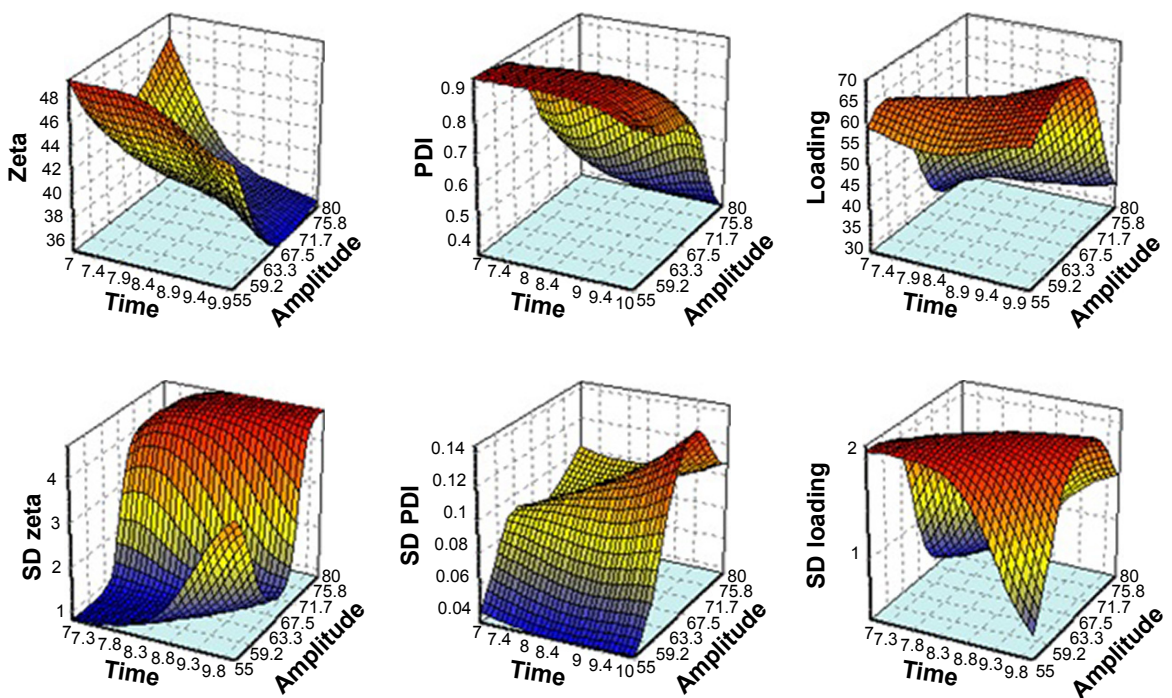

Figure 4 Response surface plots of the combined effect of (A) amplitude of sonication and drug concentration on size, zeta potential, PDI, and loading along with SD and (B) amplitude of sonication and time on size, zeta potential, PDI, and loading along with SD.

Abbreviation: PDI, polydispersity index.

the inputs. In this study, the relative effects of the amount of drug, probe amplitude, and sonication time demonstrated their profound influence on the properties of the nanoformulations. The previous findings have reported the probe amplitude and sonication time as the critical factors for the properties of nanoformulation. However, the amount of polymer, in this study, affected blandly contrarily to a previous report where the polymer was reported to be critical for the properties of nanoparticles. ${ }^{41}$

Applicability of ANN, particularly with the sparse data has already been demonstrated. ${ }^{43}$ In the present study, the above fact was supported as optimization was achievable even with a smaller number of experimentation (ie, merely with merely with five formulations). Based on the factors and response data, ANN generated five sets of predicted levels of the factors and sonication conditions for optimized nanoparticles. Set 1 given in Table 4 was selected as the composition and sonication conditions for the development of optimized DOX nanoformulation. According to set 1, the amplitude of $57.50 \%$ was round off to $60 \%$. The optimized (validated) formulation was characterized in terms of particle size, PDI, zeta potential, percentage of drug loading, morphology, FTIR, XRD, in vitro release kinetics, cellular uptake, in vitro cytotoxicity, and biodistribution. The properties of the optimized formulation were in close agreement to the predicted properties (Table 4).

The average size of optimized DOX-GCPQ nanoformulation was observed to be $73 \mathrm{~nm}$ by AFM, may be
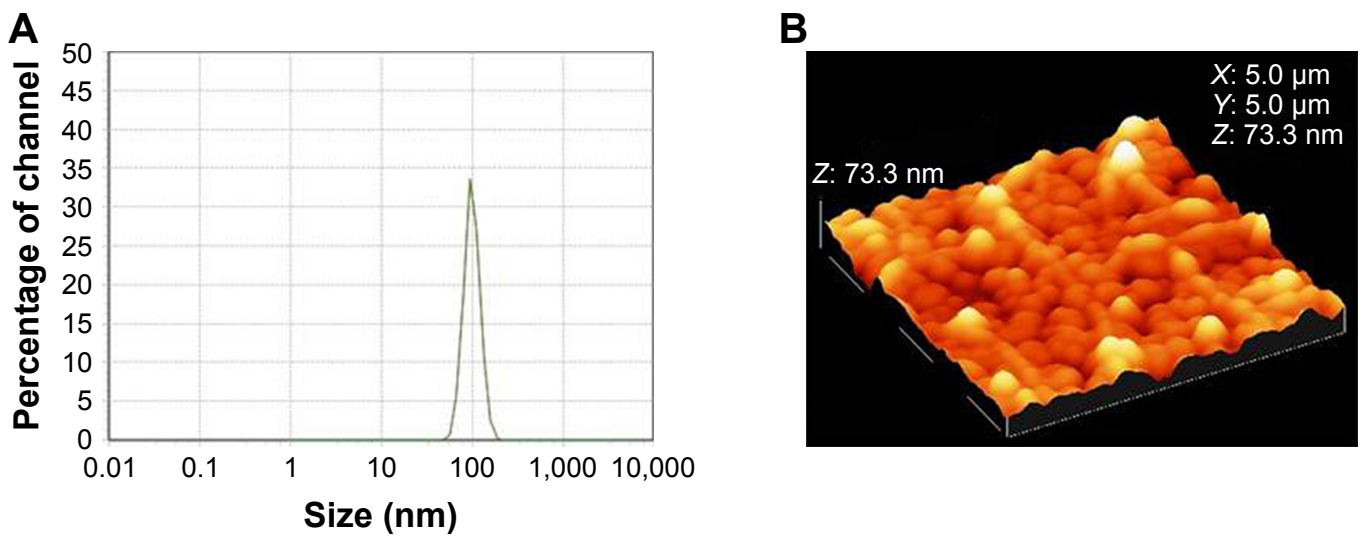

Figure 5 DOX-GCPQ.

Notes: (A) DLS plots. (B) AFM image.

Abbreviations: AFM, atomic force microscopy; DLS, dynamic light scattering; DOX, doxorubicin; GCPQ, quaternary ammonium palmitoyl glycol chitosan. 


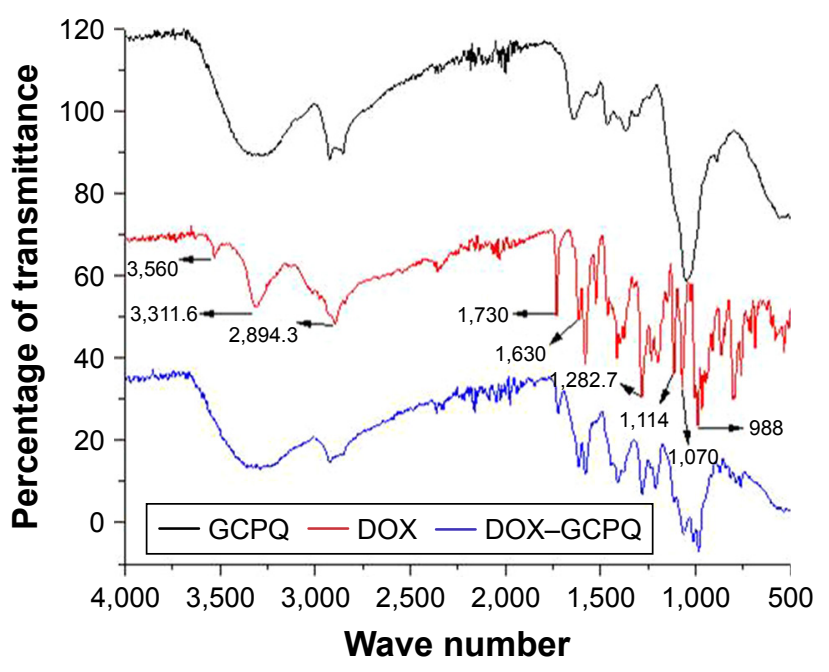

Figure 6 FTIR spectra of GCPQ, DOX and DOX-GCPQ.

Abbreviations: DOX, doxorubicin; FTIR, Fourier transformed infrared; GCPQ, quaternary ammonium palmitoyl glycol chitosan.

smaller than that measured by nano-sizer probably due to shrinkage and collapse of particle occurred during sample preparation. ${ }^{44}$ Hydrodynamic size measured by nano-sizer is of swollen particles in solution, which gives the diameter of nanoparticle with the liquid layer around the particle while AFM images particles, which are spread and dried on surface. ${ }^{45}$ Thus, the hydrodynamic diameter is larger than the actual diameter of nanoparticle measured by other techniques where particle size is determined for the dried sample. ${ }^{46}$ The zeta charge on particle was $-28 \mathrm{mV}$, the PDI was $<0.3$, and loading was $80 \%$. The particles were compact and spherical with smooth surface, and presented homogeneous population.

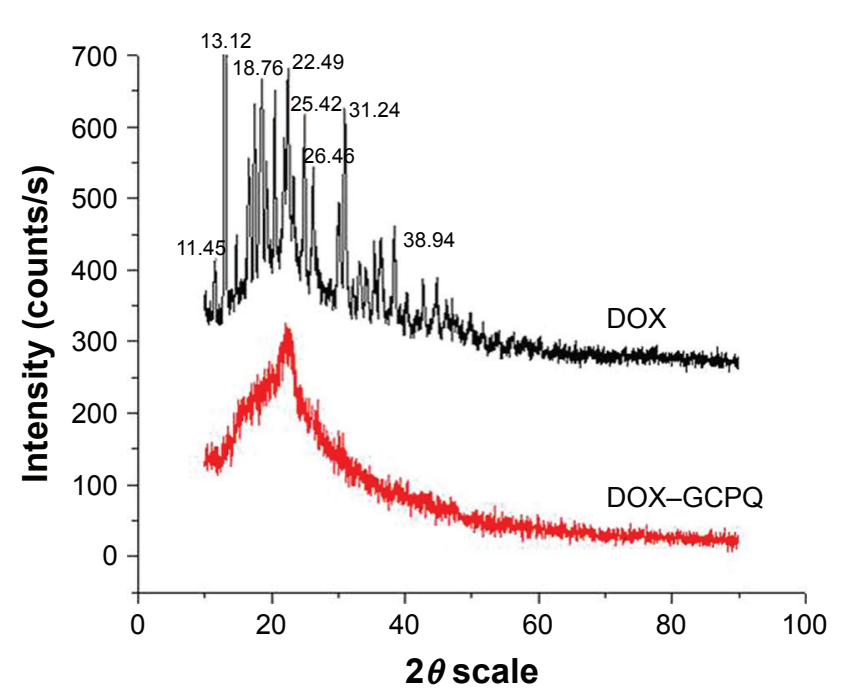

Figure 7 X-Ray diffractometer spectra of DOX and DOX-GCPQ formulation. Abbreviations: DOX, doxorubicin; GCPQ, quaternary ammonium palmitoyl glycol chitosan.

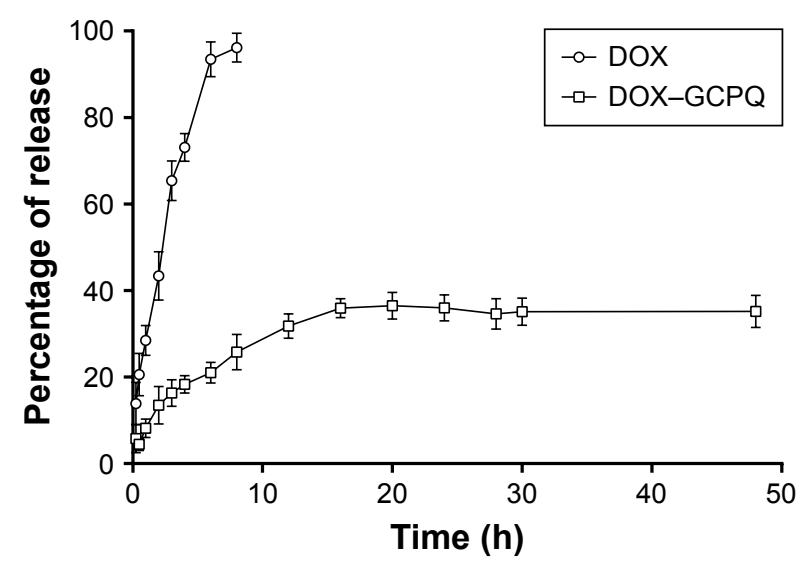

Figure 8 In vitro drug release of DOX and DOX-GCPQ nanoformulation in phosphate buffer $\left(\mathrm{pH} \mathrm{7.4)}\right.$ ) at $37^{\circ} \mathrm{C}$.

Abbreviations: DOX, doxorubicin; GCPQ, quaternary ammonium palmitoyl glycol chitosan.

In the current study, the comparative FTIR of DOX and DOX-GCPQ indicated involvement of alcoholic $\mathrm{OH}$ groups of DOX in hydrogen bonding with the GCPQ polymer. The decrease in peak intensity of carbonyl and ether groups in FTIR also indicated hydrogen bonding due to the polarity of such groups and the H-bonding between GCPQ and DOX resulted in stable nanoformulation. FTIR analysis also showed alteration in chemical structure of DOX in nanoformulation, suggesting altered state of drug from crystalline (when free) to amorphous form (when in nanoformulation). This amorphous state of DOX in nanoformulation was also supported by XRD study. XRD spectra

Table 5 In vitro drug release data modeling of DOX-GCPQ nanoformulation against DOX

\begin{tabular}{|l|l|l|l|}
\hline \multirow{2}{*}{ Kinetic model } & $\begin{array}{l}\text { Selection } \\
\text { criteria }\end{array}$ & DOX & DOX-GCPQ \\
\hline \multirow{2}{*}{ Zero order } & $R^{2}$ & 0.76162 & 0.844 \\
\cline { 2 - 4 } & AIC & 126.94 & 80.84 \\
\hline \multirow{3}{*}{ Higuchi } & $R^{2}$ & 0.900 & 0.900 \\
\cline { 2 - 4 } & AIC & 72.17 & 74.17 \\
\hline \multirow{2}{*}{ Korsmeyer-Peppas } & $R^{2}$ & 0.9608 & 0.982 \\
\cline { 2 - 4 } & AIC & 99.84 & 48.41 \\
\hline \multirow{2}{*}{ Hixson-Crowell } & $R^{2}$ & 0.994 & 0.9943 \\
\cline { 2 - 4 } & AIC & 33.06 & 33.06 \\
\hline \multirow{2}{*}{ Weibull } & $R^{2}$ & 0.9739 & 0.883 \\
\cline { 2 - 4 } & AIC & 71.92 & 76.52 \\
\hline Quadratic & $R^{2}$ & 0.996 & 0.997 \\
\cline { 2 - 4 } & AIC & 68.00 & 25.54 \\
\hline & $R^{2}$ & 0.9812 & 0.9842 \\
\cline { 2 - 4 } & AIC & 47.43 & 48.47 \\
\hline
\end{tabular}

Abbreviations: AIC, Akaike information criterion; DOX, doxorubicin; GCPQ, quaternary ammonium palmitoyl glycol chitosan. 

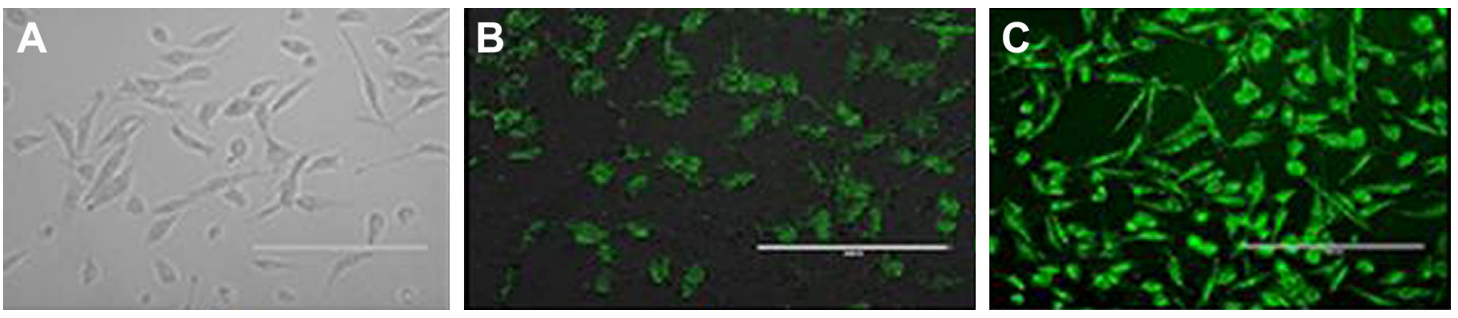

Figure 9 Cellular uptake and intracellular localization of DOX and DOX-GCPQ nanoformulations in human RD cells observed by fluorescence microscopy of (A) untreated cells, (B) fluorescence of internalized DOX inside RD cells, and (C) fluorescence of internalized DOX-GCPQ inside RD cells.

Note: Magnification 40x.

Abbreviations: DOX, doxorubicin; GCPQ, quaternary ammonium palmitoyl glycol chitosan; RD, rhabdomyosarcoma.

showed that the peaks observed for crystalline DOX were disappeared in case of DOX-GCPQ nanoformulation. ${ }^{47}$ This observation also supported a successful encapsulation of DOX inside polymer.

All the drug (100\%) was available in the dissolution media from free DOX in $10 \mathrm{~h}$, which was consistent with the previous findings. ${ }^{48} \mathrm{DOX}$ release from GCPQ was studied up to $48 \mathrm{~h}$ in accordance with the previous studies. The DOX release for GCPQ nanocarrier was found to be $35 \%$ until up to $48 \mathrm{~h}$ in this study, which was in line with the previous studies where the release was noted around $40 \%-50 \%{ }^{49}$ In vitro release study of DOX from DOX-GCPQ nanoformulation at $\mathrm{pH} 7.4$ demonstrated pattern comparable to that of the slow release since polymeric nano-delivery systems, which were designed to deliver the drug at target site via blood circulation with longer circulation time and lesser drug leakage resulted in enhanced targeting of drug with enhanced efficacy. ${ }^{48}$ Kinetic modeling of release kinetics demonstrated that DOX-GCPQ nanoformulation followed Weibull model. The $b$ value in Weibull model is indicative of drug release and determines the progression of the shape of dissolution curve. The value of $b>1$ produces sigmoidal release curve with a turning point in the initial phase, $b<1$ produces steeper increase, and $b=1$ presents exponential rise in the release curve. ${ }^{30,48}$ In this study, the $b$ value, 0.636 led to a steeper increase in the drug release curve without a sigmoid portion.
Diffusion exponential value $(n)$ of Korsmeyer-Peppas equation was used to determine drug release mechanism. Values of $n$ between 0 and 0.5 indicate that release is Fickan diffusion, and values between 0.5 and 1 indicate anomalous release due to the coupling of diffusion and erosion. ${ }^{50}$ The $n$ value of 0.59 supported a sustained drug release mechanism, which could be attributed to the hydrogen bonding between DOX and GCPQ polymer in the nanoformulation. When the release profiles of free DOX and DOX-GCPQ nanoformulation were compared, the values of dissimilarity factor $\left(f_{1}\right), 282.37$, and similarity factor $\left(f_{2}\right), 14.82$, were beyond the stipulated $\mathrm{f}_{1}$ and $\mathrm{f}_{2}$ values, ie, $0-15$ and $50-100$, respectively, to declare the similar release profiles. ${ }^{51}$

Enhanced in vitro cellular uptake of DOX-GCPQ nanoformulation as compared to the free DOX was observed that could be attributed to several reasons. The mean particle size of the present anionic DOX-GCPQ nanoformulation was below $100 \mathrm{~nm}$, ie, was $73 \mathrm{~nm}$, thus showing enhanced in vitro cellular uptake. Enhanced DOX-GCPQ internalization should present higher cytotoxicity secondary to caveolae-mediated endocytosis followed by the anionic DOX nanoformulation in comparison to the simple diffusion followed by free DOX..${ }^{52}$ In caveolae-mediated endocytosis, nanoparticles are not substrate for degradative action of lysosome ${ }^{53}$ unlike when they follow simple diffusion, thus resulting into a better accumulation of therapeutic moiety at target
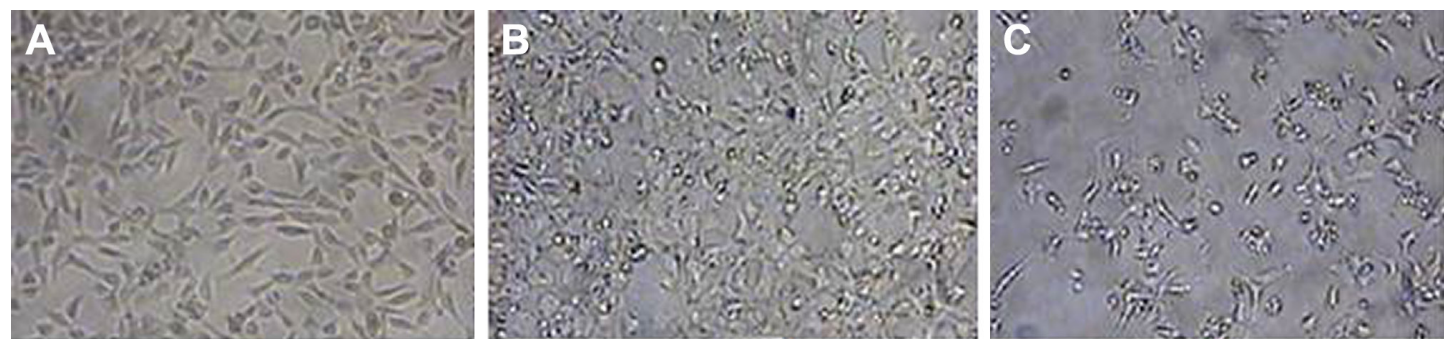

Figure 10 In vitro cytotoxicity DOX and DOX-GCPQ nanoformulation in human RD cells observed by inverted microscope after $24 \mathrm{~h}$ of incubation.

Notes: (A) Untreated RD cells. (B) Cell viability with DOX. (C) Cell viability with DOX-GCPQ. Magnification 40X.

Abbreviations: DOX, doxorubicin; GCPQ, quaternary ammonium palmitoyl glycol chitosan; RD, rhabdomyosarcoma. 


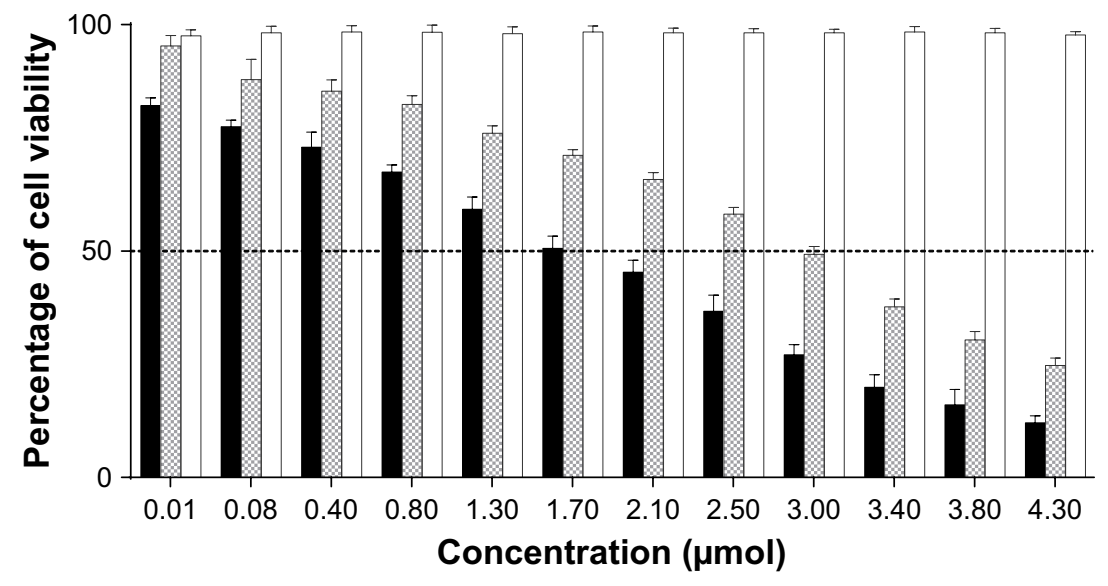

DOX $\quad$ DOX-GCPQ $\square \mathrm{GCPQ}$

Figure I I Cell viability of DOX and DOX-GCPQ against human RD cells.

Note: All cells were incubated for $24 \mathrm{~h}$, and cell viability was determined by MTT assay $(P<0.05)$.

Abbreviations: DOX, doxorubicin; GCPQ, quaternary ammonium palmitoyl glycol chitosan; RD, rhabdomyosarcoma.

site with enhanced efficacy. ${ }^{30}$ In the caveolae-dependent endocytic pathway, the caveolae 3 isoform, a muscle-specific protein, ${ }^{53}$ is responsible for more efficient internalization of nanomaterial as compared to simple diffusion. ${ }^{53}$ Furthermore, the anionic nanoformulation may be endocytosed through interaction with positive sites of proteins in membrane and highly captured by cells due to its repulsive interaction with negatively charged cell surface as reported earlier. ${ }^{54}$ Besides the above factors, the size of nanoparticle less than $100 \mathrm{~nm}$ also shows the efficient nanoformulation-mediated
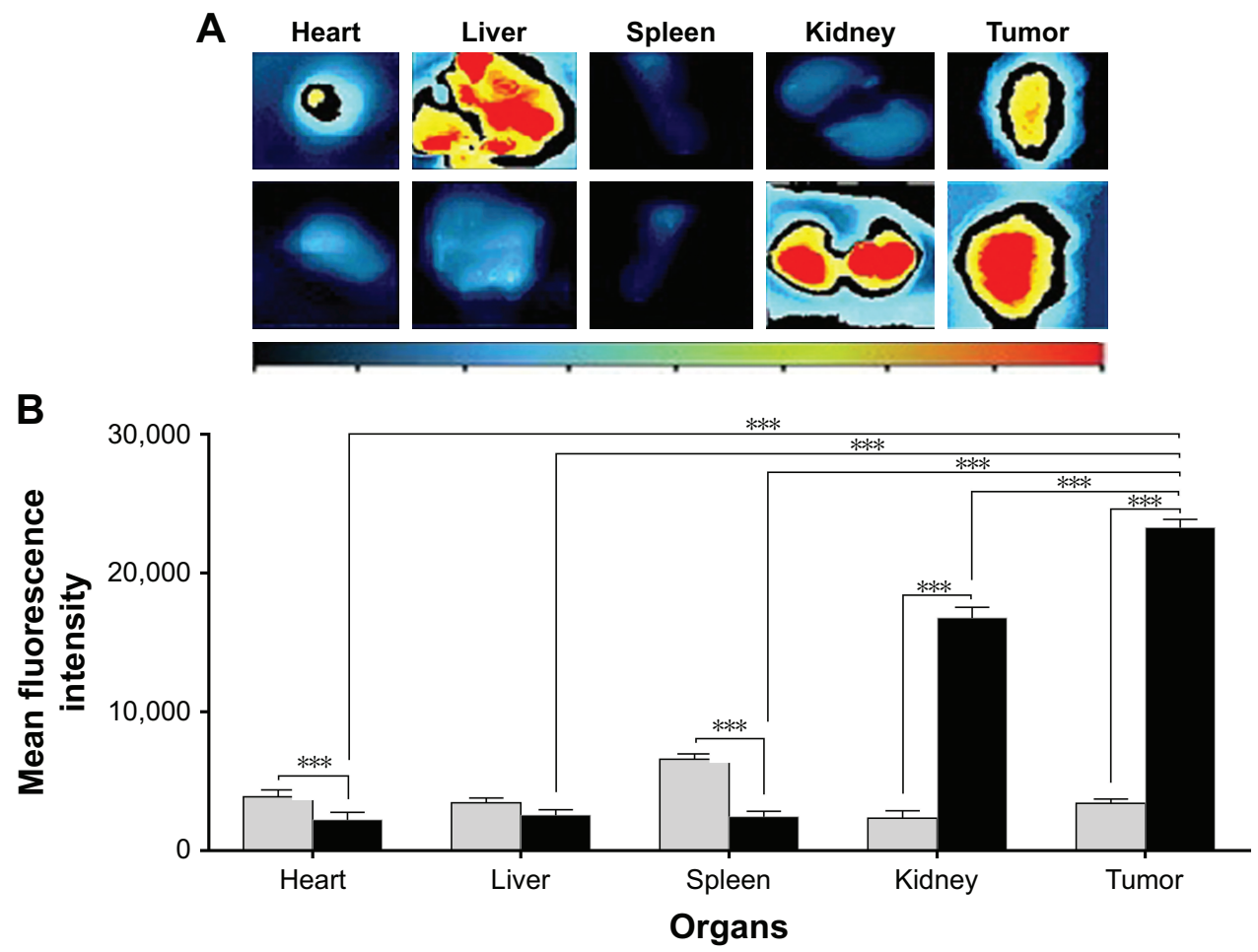

$\square$ Free DOX $\quad \mathbf{D O X}-\mathrm{GCPQ}$

Figure 12 Biodistribution of intravenously injected DOX and DOX-GCPQ in skin tumor bearing mice.

Notes: (A) Representative fluorescent images of tumor and excised organs $24 \mathrm{~h}$ after injection. (B) Quantitative fluorescent intensity of excised organ and tumor ( $P<0.05)$. $* * * P<0.0001$.

Abbreviations: DOX, doxorubicin; GCPQ, quaternary ammonium palmitoyl glycol chitosan. 
endocytosis by cells. ${ }^{55}$ Cytotoxicity experiments were conducted at 24,48 , and $72 \mathrm{~h}$ of incubation of cells with all concentrations of DOX and DOX-GCPQ. ${ }^{56,57}$ Since the best cytotoxic results were obtained after $48 \mathrm{~h}$, this time was selected to calculate $\mathrm{IC}_{50}$. The in vitro interaction of DOX-GCPQ nanoformulation with RD cell line showed significant cytotoxic effect as compared to free DOX and GCPQ polymer alone $(P<0.05)$ due to more internal localization of DOX-GCPQ. Thus, DOX-GCPQ showed lower $\mathrm{IC}_{50}$ value demonstrating its cytotoxic effect at lower dose when given as DOX-GCPQ as compared to DOX alone.

The particle size and the anionic nature of the DOXGCPQ nanoformulation impart the colloidal stability and also refrain it from interacting with mononuclear phagocytic system, up-taken nonspecifically by phagocytic system and opsonization, thus leading to improved circulation time and nonspecific distribution in healthy tissue. ${ }^{58}$ Higher colloidal stability and kinetically low dissociation allow the DOXGCPQ nanoformulation to circulate in the blood for longer time and thus, significantly increase its accumulation at the target site due to enhanced permeation by passive targeting and retention effect. ${ }^{40}$ Furthermore, slow drug release from nanoformulation is advantageous to localize cytotoxic drug to tumor site. ${ }^{48}$ Henceforth, DOX-GCPQ nanoformulation showed significant improvement in tumor accumulation as compared to the free DOX. The localization of drug in heart, liver, and spleen was significantly lower, in accordance with reported previously where GCPQ's intravenous distribution kinetics avoided uptake by liver and spleen. ${ }^{17}$ However, besides tumor, in the present study, the DOX nanoformulation was also significantly accumulated in kidney as compared to free DOX whose primary route of metabolism is liver. ${ }^{59}$ Observation of higher concentration of DOX-GCPQ in kidney might be ascribed to a changed biodistribution pattern or excretion of DOX-GCPQ via urinary route instead of biliary route reported for free DOX excretion, ${ }^{59}$ though confirmation is required through detailed biodistribution and elimination studies, respectively. Furthermore, it warrants the assessment of organ-specific toxicity of the developed formulation.

DOX encapsulation in biocompatible nano-drug delivery dosage form while effectively targeting anticancer drug to tumor both actively and passively can minimize its adverse effects, ${ }^{60}$ particularly the cardiotoxicity by virtue of its lower accumulation in heart. Controlled drug delivery system with smooth drug plasma levels over longer period of time also reduces side effects and enhances efficacy leading to better patient compliance. ${ }^{61}$
The significantly enhanced accumulation of DOX-GCPQ in tumor site has been associated with higher fluorescence signal than in other organs as compared to free drug. This method of fluorescence measurement is qualitative and semiquantitative, which presents relative uptake by organs as reported earlier. ${ }^{8,39,44,62}$ The developed DOX-GCPQ nanoformulation has demonstrated simultaneous possibility of an in vivo localization in the tumor site and optical imaging of the tumor; thus, it can be explored for its theranostic potential after detailed kinetics and in vivo imaging studies.

\section{Conclusion}

DOX-GCPQ amphiphilic polymeric nanoformulation showed accumulation in skin tumor bearing mice by optical imaging. The study enables noninvasive evaluation and in vivo biodistribution of doxorubicin nanoformulation via fluorescence imaging. The enhanced cellular uptake, desirable small particle size, sustained drug release, and ability to target tumor site passively may make it efficient nanotheranostic system.

\section{Acknowledgments}

This work is funded by PAK-NORWAY Institutional Cooperation Program, PK3004, COMSTECH-TWAS project Reference number (12-198 RG/PHA/AS_C-UNESCO FR: 3240270874) and Pakistan Science Foundation, PSF/Res/ C-NILOP/Med (330). The authors are thankful to Prof Ijeoma Uchegbu and Prof Andreas G Schatzlein, School of Pharmacy, University College London, for their analytical support in the synthesis and characterization of GCPQ.

\section{Disclosure}

The authors report no conflicts of interest in this work.

\section{References}

1. Lee VH, Robinson JR. Controlled Drug Delivery: Fundamentals and Applications. New York: Marcel Dekker; 1987.

2. Deepa K, Singha S, Panda T. Doxorubicin nanoconjugates. J Nanosci Nanotechnol. 2014;14(1):892-904.

3. Danesi R, Fogli S, Gennari A, Conte P, Del Tacca M. Pharmacokineticpharmacodynamic relationships of the anthracycline anticancer drugs. Clin Pharmacokinet. 2002;41(6):431-444

4. Outomuro D, Grana DR, Azzato F, Milei J. Adriamycin-induced myocardial toxicity: new solutions for an old problem? Int J Cardiol. 2007; 117(1):6-15.

5. Hilger RA, Richly H, Grubert M, et al. Pharmacokinetics (PK) of a liposomal encapsulated fraction containing doxorubicin and of doxorubicin released from the liposomal capsule after intravenous infusion of Caelyx/ Doxil. Int J Clin Pharmacol Ther. 2005;43(12):588-589.

6. Bharali DJ, Mousa SA. Emerging nanomedicines for early cancer detection and improved treatment: current perspective and future promise Pharmacol Ther. 2010;128(2):324-335.

7. Levenson V. Biomarkers for early detection of breast cancer: what, when, and where? Biochim Biophys Acta Gen Subj. 2007;1770(6):847-856. 
8. Chen H, Zhen Z, Todd T, Chu PK, Xie J. Nanoparticles for improving cancer diagnosis. Mater Sci Eng R Rep. 2013;74(3):35-69.

9. Chidambaram M, Manavalan R, Kathiresan K. Nanotherapeutics to overcome conventional cancer chemotherapy limitations. $J$ Pharm Pharm Sci. 2011;14(1):67-77.

10. Wadajkar AS, Menon JU, Tsai YS, et al. Prostate cancer-specific thermoresponsive polymer-coated iron oxide nanoparticles. Biomaterials. 2013;34(14):3618-3625.

11. Papahadjopoulos D, Allen TM, Gabizon A, et al. Sterically stabilized liposomes: improvements in pharmacokinetics and antitumor therapeutic efficacy. Proc Natl Acad Sci U S A. 1991;88(24):11460-11464.

12. Lasic DD, Martin FJ, Gabizon A, Huang SK, Papahadjopoulos D. Sterically stabilized liposomes: a hypothesis on the molecular origin of the extended circulation times. Biochim Biophys Acta. 1991;1070(1):187-192.

13. Liu Y, Miyoshi H, Nakamura M. Nanomedicine for drug delivery and imaging: a promising avenue for cancer therapy and diagnosis using targeted functional nanoparticles. Int J Cancer. 2007;120(12):2527-2537.

14. Slingerland M, Guchelaar HJ, Gelderblom H. Liposomal drug formulations in cancer therapy: 15 years along the road. Drug Discov Today. 2012;17(3-4):160-166.

15. Masood F. Polymeric nanoparticles for targeted drug delivery system for cancer therapy. Mater Sci Eng C. 2016;60(1):569-578.

16. Emami J, Rezazadeh M, Hasanzadeh F, et al. Development and in vitro/ in vivo evaluation of a novel targeted polymeric micelle for delivery of paclitaxel. Int J Biol Macromol. 2015;80:29-40.

17. Lalatsa A, Schätzlein AG, Mazza M, Le TB, Uchegbu IF. Amphiphilic poly(L-amino acids) - new materials for drug delivery. $J$ Control Release. 2012;161(2):523-536.

18. Kim J, Lee JE, Lee SH, et al. Designed fabrication of a multifunctional polymer nanomedical platform for simultaneous cancer-targeted imaging and magnetically guided drug delivery. Adv Mater. 2008;20(3): $478-483$.

19. Kunjachan S, Ehling J, Storm G, Kiessling F, Lammers T. Noninvasive imaging of nanomedicines and nanotheranostics: principles, progress, and prospects. Chem Rev. 2015;115(19):10907-10937.

20. Vollrath A, Schubert S, Schubert US. Fluorescence imaging of cancer tissue based on metal-free polymeric nanoparticles - a review. J Mater Chem B. 2013;1(15):1994-2007.

21. Nguyen HN, Hoang TMN, Mai TTT, et al. Enhanced cellular uptake and cytotoxicity of folate decorated doxorubicin loaded PLA-TPGS nanoparticles. Adv Nat Sci Nanosci Nanotechnol. 2015;6(2):025005.

22. Li X, Hirsh DJ, Cabral-Lilly D, et al. Doxorubicin physical state in solution and inside liposomes loaded via a pH gradient. Biochim Biophys Acta. 1998;1415(1):23-40.

23. Thanoo BC, Sunny MC, Jayakrishnan A. Cross-linked chitosan microspheres: preparation and evaluation as a matrix for the controlled release of pharmaceuticals. J Pharm Pharmacol. 1992;44(4):283-286.

24. Shiraishi S, Imai T, Otagiri M. Controlled release of indomethacin by chitosan-polyelectrolyte complex: optimization and in vivo/in vitro evaluation. J Control Release. 1993;25(3):217-225.

25. ICH [homepage on the Internet]; 2018. Available from: http://www.ich. org/products/guidelines/safety/article/safety-guidelines.html. Accessed September 13, 2018.

26. Raza A, de la Fuente M, Uchegbu IF, Schätzlein A. Modified glycol chitosan nanocarriers carry hydrophobic materials into tumours. Paper presented at: Nanotechnology 2010: Bio Sensors, Instruments, Medical, Environment and Energy-Technical Proceedings of the 2010 NSTI Nanotechnology Conference and Expo, NSTI-Nanotech 2010.

27. Uchegbu IF, Sadiq L, Arastoo M, et al. Quaternary ammonium palmitoyl glycol chitosan - a new polysoap for drug delivery. Int J Pharm. 2001;224(1-2):185-199.

28. Javed I, Hussain SZ, Ullah I, et al. Synthesis, characterization and evaluation of lecithin-based nanocarriers for the enhanced pharmacological and oral pharmacokinetic profile of amphotericin B. J Mater Chem B. 2015;3(42):8359-8365.

29. Lokhandwala H, Deshpande A, Deshpande S. Kinetic modeling and dissolution profiles comparison: an overview. Int J Pharm Bio Sci. 2013;4(1):728-773
30. Kou L, Sun J, Zhai Y, He Z. The endocytosis and intracellular fate of nanomedicines: Implication for rational design. Asian J Pharm Sci. 2013; $8(1): 1-10$

31. Li F, Zheng C, Xin J, et al. Enhanced tumor delivery and antitumor response of doxorubicin-loaded albumin nanoparticles formulated based on a Schiff base. Int J Nanomedicine. 2016;11:3875.

32. Wang L, Zhang J, Song M, et al. A shell-crosslinked polymeric micelle system for $\mathrm{pH} /$ redox dual stimuli-triggered DOX on-demand release and enhanced antitumor activity. Colloids Surf B Biointerfaces. 2017;152:1-11.

33. Mosmann T. Rapid colorimetric assay for cellular growth and survival: application to proliferation and cytotoxicity assays. J Immunol Methods. 1983;65(1-2):55-63.

34. Abel EL, Angel JM, Kiguchi K, Digiovanni J. Multi-stage chemical carcinogenesis in mouse skin: fundamentals and applications. Nat Protoc. 2009;4(9):1350-1362.

35. Jin $\mathrm{ZH}$, Jin MJ, Jiang CG, et al. Evaluation of doxorubicin-loaded $\mathrm{pH}$-sensitive polymeric micelle release from tumor blood vessels and anticancer efficacy using a dorsal skin-fold window chamber model. Acta Pharmacol Sin. 2014;35(6):839-845.

36. Anders CK, Adamo B, Karginova O, et al. Pharmacokinetics and efficacy of PEGylated liposomal doxorubicin in an intracranial model of breast cancer. PLoS One. 2013;8(5):e61359.

37. Gustafson DL, Merz AL, Long ME. Pharmacokinetics of combined doxorubicin and paclitaxel in mice. Cancer Lett. 2005;220(2):161-169.

38. Sajja HK, East MP, Mao H, Wang YA, Nie S, Yang L. Development of multifunctional nanoparticles for targeted drug delivery and noninvasive imaging of therapeutic effect. Curr Drug Discov Technol. 2009; 6(1):43-51.

39. Shalviri A, Cai P, Rauth AM, Henderson JT, Wu XY. Evaluation of new bi-functional terpolymeric nanoparticles for simultaneous in vivo optical imaging and chemotherapy of breast cancer. Drug Deliv Transl Res. 2012;2(6):437-453.

40. Uchegbu IF, Carlos M, McKay C, Hou X, Schätzlein AG. Chitosan amphiphiles provide new drug delivery opportunities. Polym Int. 2014; 63(7):1145-1153.

41. Esmaeilzadeh-Gharedaghi E, Faramarzi MA, Amini MA, Rouholamini Najafabadi A, Rezayat SM, Amani A. Effects of processing parameters on particle size of ultrasound prepared chitosan nanoparticles: an Artificial Neural Networks Study. Pharm Dev Technol. 2012;17(5): 638-647.

42. Agatonovic-Kustrin S, Beresford R. Basic concepts of artificial neural network (ANN) modeling and its application in pharmaceutical research. J Pharm Biomed Anal. 2000;22(5):717-727.

43. Bukhari NI, Julianto T, Valente Pereira RE. Computer-aided prediction of cefotaxime sodium stability in aqueous solution at different $\mathrm{pH}$ from sparse data. Latin Am J Pharm. 2018;37(3):571-578.

44. Zhang J, Sun Y, Tian B, et al. Multifunctional mesoporous silica nanoparticles modified with tumor-shedable hyaluronic acid as carriers for doxorubicin. Colloids Surf B Biointerfaces. 2016;144: 293-302.

45. Qi J, Yao P, He F, Yu C, Huang C. Nanoparticles with dextran/chitosan shell and BSA/chitosan core-Doxorubicin loading and delivery. Int $J$ Pharm. 2010;393(1-2):177-185.

46. Eaton P, Quaresma P, Soares C, et al. A direct comparison of experimental methods to measure dimensions of synthetic nanoparticles. Ultramicroscopy. 2017;182:179-190.

47. Sharma R, Bisen D, Shukla U, Sharma B. X-ray diffraction: a powerful method of characterizing nanomaterials. Recent Res Sci Technol. 2012;4(8):77-79.

48. Liu G, Jin Q, Liu X, Lv L, Chen C, Ji J. Biocompatible vesicles based on PEO-b-PMPC/ $\alpha$-cyclodextrin inclusion complexes for drug delivery. Soft Matter. 2011;7(2):662-669.

49. Yuan A, Wu J, Song C, et al. A novel self-assembly albumin nanocarrier for reducing doxorubicin-mediated cardiotoxicity. J Pharm Sci. 2013; 102(5):1626-1635.

50. Ramteke K, Dighe P, Kharat A, Patil S. Mathematical models of drug dissolution: a review. Sch Acad J Pharm. 2014;3(5):388-396. 
51. Osei-Asare C, Kipo S, Ofori-Kwakye K, Boakye-Gyasi M. Comparative in vitro dissolution of commercially available sustained release nifedipine tablet brands in the Kumasi Metropolis, Ghana. J Appl Pharm Sci. 2015:54-60.

52. Bannunah AM, Vllasaliu D, Lord J, Stolnik S. Mechanisms of nanoparticle internalization and transport across an intestinal epithelial cell model: effect of size and surface charge. Mol Pharm. 2014;11(12): 4363-4373.

53. Benmerah A, Lamaze C. Clathrin-coated pits: vive la différence? Traffic. 2007;8(8):970-982.

54. Yeung T, Gilbert GE, Shi J, Silvius J, Kapus A, Grinstein S. Membrane phosphatidylserine regulates surface charge and protein localization. Science. 2008;319(5860):210-213.

55. Singh R, Lillard JW. Nanoparticle-based targeted drug delivery. Exp Mol Pathol. 2009;86(3):215-223.

56. Shafiu Kamba A, Ismail M, Tengku Ibrahim TA, Zakaria ZAB, Hassan Gusau L. In vitro ultrastructural changes of MCF-7 for metastasise bone cancer and induction of apoptosis via mitochondrial cytochrome $\mathrm{C}$ released by $\mathrm{CaCO}_{3} /$ Dox nanocrystals. Biomed Res Int. 2014;2014(3):1-14.
57. Cao N, Feng SS. Doxorubicin conjugated to D-alpha-tocopheryl polyethylene glycol 1000 succinate (TPGS): conjugation chemistry, characterization, in vitro and in vivo evaluation. Biomaterials. 2008; 29(28):3856-3865.

58. Blanco E, Shen H, Ferrari M. Principles of nanoparticle design for overcoming biological barriers to drug delivery. Nat Biotechnol. 2015; 33(9):941-951.

59. Wang Y, Mei X, Yuan J, Lu W, Li B, Xu D. Taurine zinc solid dispersions attenuate doxorubicin-induced hepatotoxicity and cardiotoxicity in rats. Toxicol Appl Pharmacol. 2015;289(1):1-11.

60. Piktel E, Niemirowicz K, Wątek M, Wollny T, Deptuła P, Bucki R Recent insights in nanotechnology-based drugs and formulations designed for effective anti-cancer therapy. J Nanobiotechnology. 2016; 14(1):39.

61. Jayakumar R, Nair A, Rejinold NS, Maya S, Nair SV. Doxorubicinloaded $\mathrm{pH}$-responsive chitin nanogels for drug delivery to cancer cells. Carbohydr Polym. 2012;87(3):2352-2356.

62. Hwang JY, Park J, Kang BJ, et al. Multimodality imaging in vivo for preclinical assessment of tumor-targeted doxorubicin nanoparticles. PLoS One. 2012;7(4):e34463.
International Journal of Nanomedicine

\section{Publish your work in this journal}

The International Journal of Nanomedicine is an international, peerreviewed journal focusing on the application of nanotechnology in diagnostics, therapeutics, and drug delivery systems throughout the biomedical field. This journal is indexed on PubMed Central, MedLine, CAS, SciSearch $®$, Current Contents $\AA /$ Clinical Medicine,

\section{Dovepress}

Journal Citation Reports/Science Edition, EMBase, Scopus and the Elsevier Bibliographic databases. The manuscript management system is completely online and includes a very quick and fair peer-review system, which is all easy to use. Visit http://www.dovepress.com/ testimonials.php to read real quotes from published authors. 